African Crop Science Journal by African Crop Science Society is licensed under a Creative Commons Attribution 3.0 Uganda License. Based on a work at www.ajol.info/ and www.bioline.org.br/cs DOI: http://dx.doi.org/10.4314/acsj.v24i2.3

\title{
GENETIC EVALUATION OF SEED TRAITS FROM INTRASPECIFIC CROSSING OF GENETICALLY DISTINCT WATERMELON VARIETIES
}

\author{
K. ADJOUMANI, S.B. BONY, G.K. KOFFI, L.C. KOUONON, F.K. BROU and R. SIÉ \\ Université Nangui-Abrogoua, Laboratoire de Biologie et Amélioration des Productions Végétales, 02 BP 801 \\ Abidjan 02, Côte d'Ivoire \\ Corresponding author: adjoumani.koffi@yahoo.fr
}

(Received 30 October, 2015; accepted 16 May, 2016)

\begin{abstract}
Citrullus lanatus (Thunb.) Matsumura and Nakai (Cucurbitaceae) is an important cucurbit crop worldwide. Global production of watermelon is about 90 million metric tonnes per annum, making it among the top five most consumed fresh fruits. The objective of this study was to evaluate seed variability in different segregating populations, and determine heritability of traits of watermelon. Interspecific crosses were made between two cultivars of $C$. lanatus (Bebu and Wlêwlê Small Seeds (WSS) were performed at Research Station of Nangui Abrogoua University in Abidjan, Côte d'Ivoire. There was wide variability between parental, $\mathrm{F}_{1}, \mathrm{BC}_{1}$ (first generation of back-crossing) and $\mathrm{F}_{2}$ seeds. Seeds of all hybrid populations were intermediate versus those of the parents. Also, crossing did not affect $\mathrm{F}_{1}$ and $\mathrm{F}_{2}$ seed characters, but affected those of $\mathrm{BC}_{1}$ because of maternal effects. Thus, back-crossing on Bebu cultivar produced seeds which looked like those of Bebu; while backcrossing on WSS cultivar produced seeds similar to those of WSS. Principal Component Analysis (PCA) and individuals repartitioning revealed that Bebu and WSS cultivars were genetically distinct and showed three main groups: two groups from each parental line and one from a recombinant line (hybrids). $F_{2}$ population had a wide individual's dispersion, and contained seeds of all other populations. High heritability was observed for all evaluated characters.
\end{abstract}

Key Words: Citrullus lanatus, Côte d'Ivoire, heritability, hybrids

RESUME

Citrullus lanatus (Thunb.) Matsumara et Nakai (Cucurbitacée) est une importante cucurbite dans le monde entier. La production annuelle globale est d'environ 90 million de tonne, ce qui place cette culture parmi les cinq fruits les plus consommés crus. L'objectif de cette étude est d'évaluer la variabilité des graines de différentes populations en ségrégation et de déterminer l'héritabilité des caractères chez l'espèce Citrullus lanatus. Pour cela, un croisement interspécifique entre deux cultivars de Citrullus lanatus (Bebu et Wlêwlề à petites graines (WSS)) a été effectué à la station de recherche de l'Université Nangui Abrogoua d'Abidjan (Côte d'Ivoire). Il existe une variabilité importante entre les graines parentales, $\mathrm{F}_{1}, \mathrm{BC}_{1}$ (première génération de back-cross) et $\mathrm{F}_{2}$. Les graines de toutes les populations hybrides sont intermédiaires à celles des parents. Aussi, le sens de croisement n'affecte pas les caractères des graines $\mathrm{F}_{1}$ et $\mathrm{F}_{2}$ mais affecte ceux des graines $\mathrm{BC}_{1}$ à cause de l'effet maternel. Ainsi, un backcross effectué sur le cultivar Bebu produit des graines semblables aux graines de Bebu tandis qu'un backcross effectué sur le cultivar WSS produit des graines semblables aux graines de WSS. L'Analyse en Composantes Principales et la répartition des individus dans le plan permettent d'observer que les cultivars Bebu et WSS sont génétiquement distincts et montrent trois principaux groupes : deux groupes de chaque type parental et un type recombinant (hybride). La population $\mathrm{F}_{2}$ a une large dispersion et se compose des graines de toutes les autres populations. Une 
forte héritabilité a été observée pour tous les caractères évalués. En somme, la variabilité des graines observées dans les populations en ségrégation chez $C$. lanatus pourrait être due à des effets génétiques

Mots Clés : Citrullus lanatus, Côte d'Ivoire, héritabilité, hybride

\section{INTRODUCTION}

Citrullus lanatus (Thunb.) Matsumura and Nakai (Cucurbitaceae) is an important cucurbit crop globally. World production of the crop is about 90 million metric tonnes annually, making it one of the top five most consumed fresh fruits. Citrullus lanatus can be found growing wild in various areas of the world, particularly in India and Mediterranean region, including Iran and Egypt (Kumar and Wehner, 2011).

In west-Africa, this traditional vegetable is perceived as potentially useful for income generation, food security (Achigan-Dako et al., 2008; Dos Santos et al., 2012) and medicinal uses (Abdel and Bamerni, 2011). Despite these numerous agronomic, social, health and economic potentials, along with its good adaptation to extremely divergent agro-ecosystems, $C$. lanatus cultivars are either missing in inbreeding programmes, neglected or underutilised (also called orphan crops). Consequently, there is lack of informations about genetics and breeding of this important crop in many African countries. Also, the volume supplied is often far short of market demand. There is need for cultivar improvement, especially for increased seed traits, particularly for countries such as Côte d'Ivoire.

In general, the traditional varieties are less productive than hybrids and improved cultivars, although they are highly adapted to specific cultivation conditions, and also a very significant repository of important genes for drought and pests resistance (Dos Santos et al., 2012).

In the last decade, the proportion of watermelon hybrids has dramatically increased in occidental countries because of the advantages of heterosis effect, improved technology and underutilisation of controlled environments (Nerson, 2007). In these regions, watermelon breeders have contributed to the development of new cultivars and understanding of the genetics of the useful traits. In population improvement, it is imperative to determine the extent of genetic variation for a trait to be improved (Dos Santos et al., 2012).

The basic idea in the study of yield variation is its partitioning into components attributable to different causes, and the relative magnitude of these components determines the genetic properties of the population (Falconer, 1989). This led to the concept of heritability, which estimates the relative contributions of the differences in genetic and non-genetic factors, to the total phenotypic variance in a population. Determining the components of variability in yield and its components will also enable us to understand the extent of environmental influence on yield, taking into consideration the fact that yield and its components are quantitative characters and are affected by environment (Tazeen et al., 2009). The objective of this research was to estimate the magnitude of the various components of variation, heritability and genetic advance in $C$. lanatus cultivars populations in Côte d'Ivoire.

\section{MATERIALS AND METHODS}

Study sites. The study was conducted in two locations; Abidjan (Research Station of Nangui Abrogoua University) and Manfla, both in Côte d'Ivoire. Abidjan is located in Lagoon District in southern Côte d'Ivoire ( $48^{\circ} 41^{\prime}$ N, $48^{\circ} 00^{\prime}$ W). The rainfall is abundant (annual mean $>2000 \mathrm{~mm}$ ) and annual mean temperature is about $28{ }^{\circ} \mathrm{C}$. Vegetation is mainly represented by the tropical rain forest, with mangrove on the coastal side (Zoro et al., 2006).

Manfla is a village closest to Gohitafla city in the Department of Zuenoula (District of Marahoue), in the west-centre of Côte d'Ivoire, between latitudes $7^{\circ} 00^{\prime} \mathrm{N}$ and $7^{\circ} 26^{\prime} \mathrm{N}$ and longitudes $6^{\circ} 00 \mathrm{~W}$ and $6^{\circ} 30^{\prime} \mathrm{W}$ (Kouassi and Zoro Bi, 2009). Rainfall is regular (annual mean of $1500 \mathrm{~mm}$ ) and annual average temperature is about $27^{\circ} \mathrm{C}$. Vegetation is mainly represented by the tropical forest. 
Plant materials. Two parental cultivars of $C$. lanatus (Bebu and Wlêwlê Small Seeds) and their offsprings $\left(\mathrm{F}_{1}, \mathrm{BC}_{1}\right.$ and $\left.\mathrm{F}_{2}\right)$ obtained from crosses were used in the study. Bebu and Wlêwlê Small Seeds (WSS) were genetically distinct. Big fruits coming from Bebu contained few big seeds and were harvested early; while small fruits coming from WSS, contained small seeds and were harvested late (Adjoumani et al., 2012). Seeds of these parental cultivars were provided for five successive self-pollination experiments, and were conserved in the Genetic Laboratory of Nangui Abrogoua University, under introduction numbers (NI) that were NI 121 for Bebu cultivar and NI 113 for WSS cultivar. Bebu and WSS were crossed and three offspring generations $\left(\mathrm{F}_{1}, \mathrm{~F}_{2}\right.$ and $\mathrm{BC}_{1}$ ) were produced.

Treatments. Before the crossings assessed in this study, purity of parental cultivars (Bebu and WSS) was obtained previously after five selfpollination generations. Then, reciprocal crosses between Bebu and WSS cultivars (Bebu오 x WSS $\delta^{\prime}$ and WSS $q \times$ Bebu $\delta^{\prime \prime}$ ) were done from March to June 2011. After these crosses and for each cross, $\mathrm{F}_{1}$ plants were produced by selecting seeds from three fruits coming from these different plants. $\mathrm{F}_{1 \mathrm{~b}}$ and $\mathrm{F}_{1 \mathrm{w}}$ were both families developed, respectively, from these reciprocal crosses. Then, from August to November 2011, parental, $\mathrm{F}_{1 \mathrm{~b}}$ and $\mathrm{F}_{1 \mathrm{w}}$ seeds were sowed in the same plot to produce $\mathrm{BC}_{1}$ and $\mathrm{F}_{2}$ seeds. From $\mathrm{F}_{1 \mathrm{~b}}$ and $\mathrm{F}_{1 \mathrm{w}}$ plants, selfpollination was performed to produce $\mathrm{F}_{2 \mathrm{~b}}$ and $\mathrm{F}_{2 \mathrm{w}}$ plants, respectively.

Crosses between $\mathrm{F}_{1 \mathrm{w}}$ plants, and each parent produced $\mathrm{BC}_{1 \mathrm{~b}}$ and $\mathrm{BC}_{1 \mathrm{w}}$ plants (respectively backcross on Bebu and WSS). All generation (parental and hybrids) seeds were sown from March to June 2012, in the same environment, at two different agro-ecological locations, namely, Manfla (savannah) and Research Station of Nangui Abrogoua University (forest). The distance between these two experimental locations (Manfla-Abidjan) was $400 \mathrm{Km}$.

In both locations, each field was $105 \mathrm{~m} \times 60 \mathrm{~m}$ and contained 32 rows, with $3 \mathrm{~m}$ between them. The number of rows in each field was two for each parental line, three for each $\mathrm{F}_{1}$ family and six for each back cross $\left(\mathrm{BC}_{1}\right)$ and $\mathrm{F}_{2}$ families. A total of 2-3 seeds of each family were planted per hole, with an intra-row spacing of $2 \mathrm{~m}$. Ten days after sowing, seedlings were separated and the most vigorous were chosen. In each location, individuals in a family were 30,60, and 180, respectively; for each parent, $\mathrm{F}_{1} \mathrm{BC}_{1}$ and $\mathrm{F}_{2}$ family. Regular weeding of the experimental fields was done during plant vegetative cycle.

Parameters evaluated. Three fruits per plant were selected to evaluate mature seed parameters. This was done as follow:

Fresh seed mass was determined as seed mass extracted directly per fruit, weighed with precision balance (Ohaus adventurer balance; 0,001 g sensibility).

Dry seed mass was determined as seeds from the same fruit dried in natural condition and weighed at regular intervals of two (2) days during two weeks with precision balance (Ohaus adventurer balance; 0,001 g sensibility), until a constant mass. Seeds were considered dry when mass stayed constant between two consecutive interval measurements. The last measurement corresponded to dry seed mass per fruit.

For 100 seeds mass, a batch of 100 dry seeds per fruit weighed with precision balance. As for percentage seed integument, for each fruit, a batch of 20 undecorticated dry seeds (seed with integument, $\mathrm{M}_{20} \mathrm{UD}$ ) was weighed. Seeds where then decorticated and weighed again to determine the mass of the bacth of 20 decorticated dry seeds (seed without integument, $\mathrm{M}_{20} \mathrm{D}$ ). Seed integument percentage was then calculated.

Seed length was determined as the distance between the seed base and its opposite apex measured on 20 seed samples extracted in three fruits per plant.

Seed width was determined as the lateral diameter measured on 20 seed samples extracted in three fruits per plant.

Statistical test. Two way analysis of variance [ANOVA 2: families (parents, $\mathrm{F}_{1 \mathrm{~b}}, \mathrm{~F}_{1 \mathrm{w}}, \mathrm{F}_{2 \mathrm{~b}}, \mathrm{~F}_{2 \mathrm{w}}, \mathrm{BC}_{1 \mathrm{~b}}$ and $\mathrm{BC}_{1 \mathrm{w}}$ ) and locations (Manfla and Abidjan)] were used to evaluate parental and hybrid performance, following seed traits with SAS software (SAS, 2006). This test evaluated variability between families, the main effects of location on family production and the interaction between families-locations. Also, Principal 
Components Analysis (PCA) was performed in order to seek variability factors and to observe individuals regrouping.

Estimating heritability and predicting selection response were then made. A design based on the measure of variance from parent and offspring was used to estimate environmental, genetic, and additive variances (Warner, 1952; Wright, 1968).

\section{RESULTS}

Seed size (length and width), seed mass and seed integument thickness varied significantly $(\mathrm{P}<$ 0.001) among families (Table 1). Bebu yielded the biggest seeds, with at least $18 \mathrm{~cm}$ and $10 \mathrm{~cm}$ length and width, respectively; while WSS yielded the smallest seeds with at least $11 \mathrm{~cm}$ and $4 \mathrm{~cm}$ for the same characters. The hybrids $\left(\mathrm{F}_{1}, \mathrm{BC}_{1}\right.$ and $\left.\mathrm{F}_{2}\right)$ seed size were intermediate to parents. Indeed, backcross on Bebu yielded big seeds neighbouring Bebus' seeds size; while backcross on WSS yielded small seeds neighbouring the seed size of WSS. $F_{2}$ seeds contained a wide distribution of seed sizes. None of them looked like parental seed, others like $\mathrm{F}_{1}$ and $\mathrm{BC}_{1}$ and others again like a new recombinants (Fig. 1).

Bebu big seeds were the heaviest; while WSS small seeds were the lightest (Table 1). Thus, spawn seed yielded by Bebu weighed $54 \mathrm{~g}$; while those of WSS weighed $24 \mathrm{~g}$; representing less than half the mass of these first ones. Upon drying, they weighed, respectively, 28 and $8 \mathrm{~g}$.

The means of offspring $\left(\mathrm{F}_{1}, \mathrm{BC}_{1}\right.$ and $\left.\mathrm{F}_{2}\right)$ seed mass were intermediate to the one of parents. Partial dominance (heavy seed phenotype/light seed phenotype) was observed because $\mathrm{F}_{1}$ hybrid seeds were intermediate to the parents and had average mass approximating the mid-parent average. $\mathrm{BC}_{1 \mathrm{~b}}$ seeds were heaviest due to the maternal effect. In fact, seed mass yielded in $\mathrm{BC}_{1}$ reflected the one of maternal parent. So, backcrossing on Bebu cultivar $\left(\mathrm{BC}_{1 \mathrm{~b}}\right)$ produced heavy seeds like the Bebu's seeds; and backcross on WSS cultivar $\left(\mathrm{BC}_{1 \mathrm{w}}\right)$ produced light seeds similar to WSS's seeds.

Bebu seed integument was quite thick compared to that of WSS. Integument thickness of offspring seeds was intermediate to the one of parents. In the hybrid populations, $\mathrm{BC}_{1 \mathrm{~b}}$ seed integument was the thickest due to the maternal effects. So, backcrossing on Bebu cultivar $\left(\mathrm{BC}_{1 \mathrm{~b}}\right)$ produced thick integuments ( 34 to $35 \%$ of $\mathrm{BC}_{1 \mathrm{~b}}$ total seed mass). Bebu's seeds integuments (36 to $37 \%$ of Bebu total seed mass); and back cross on WSS cultivar $\left(\mathrm{BC}_{1 \mathrm{w}}\right)$ produced thin seed integuments (19 to $20 \%$ of $\mathrm{BC}_{1 \mathrm{w}}$ total seed mass) as WSS's seeds (18 to $19 \%$ of WSS total seed mass). Seeds produced in $F_{1}$ had thicker integuments than $\mathrm{F}_{2}$ seeds.

Cross direction did not affect seed size, seed mass and seed integument thickness in both $\mathrm{F}_{1}$ and $\mathrm{F}_{2}$ generations, but it affected $\mathrm{BC}_{1}$ seed traits due to the maternal effects. So whatever cross direction, $F_{1 b}$ and $F_{1 w}$, and $F_{2 b}$ and $F_{2 w}$ had statistically the same seed traits. According to cross directions, seed integument thickness represented, respectively, 28.33 to $32.57 \%$ and 23.86 to $28.69 \%$ of $F_{1}$ and $F_{2}$ total seed mass according to locations. Seed integument thickness represented 34 to $35 \%$ and 19 to $20 \%$ of $\mathrm{BC}_{1 \mathrm{~b}}$ and $\mathrm{BC}_{1 \mathrm{w}}$ total seed mass, respectively.

There were significant genetic distances between populations (Table 2). Genetic distances between parental cultivars were greater than those between them and their offsprings; and between offsprings. Bebu and WSS were far genetically, and the majority of their offsprings were located between them. Besides, the genetic distance between $\mathrm{Bebu}$ and $\mathrm{BC}_{1 \mathrm{w}}$ was superior to that between Bebu and $\mathrm{BC}_{1 \mathrm{~b}}$. Likewise, the genetic distance between WSS and $\mathrm{BC}_{1 \mathrm{~b}}$ was superior to that between WSS and $\mathrm{BC}_{1 \mathrm{w}}$.

Table 3 presents relative individuals repartition from genetic distance on both sites, showing difference between Bebu and WSS individuals, $\mathrm{BC}_{1 \mathrm{~b}}$ and WSS and between $\mathrm{BC}_{1 \mathrm{w}}$ and Bebu. However, some $\mathrm{BC}_{1 \mathrm{~b}}$ and $\mathrm{BC}_{1 \mathrm{w}}$ individuals looked like, respectively, Bebu and WSS. Maternal effects were observed in those $\mathrm{BC}_{1}$ populations. All hybrid $\left(\mathrm{F}_{1}, \mathrm{BC}_{1}\right.$ and $\left.\mathrm{F}_{2}\right)$ populations had some common individuals. $\mathrm{F}_{1}$ phenotypes were observed only in $\mathrm{BC}_{1}$ and $\mathrm{F}_{2}$ populations; while those of $\mathrm{F}_{2}$ reflected all populations (parental, $\mathrm{F}_{1}$ and $\mathrm{BC}_{1}$ ).

Principal Components Analysis (PCA) indicated that variations between populations were bound to one principal factor, regardless of experimental site (Table 4). This first and main factor contributed $77.31 \%$ for Abidjan 
TABLE 1. Means \pm standard deviation of each population according to traits and locations

\begin{tabular}{|c|c|c|c|c|c|c|c|c|c|c|c|}
\hline \multirow[t]{2}{*}{ Traits } & \multirow[t]{2}{*}{ Sites } & \multicolumn{8}{|c|}{ Populations } & \multicolumn{2}{|c|}{ Statistical tests } \\
\hline & & Bebu & $\mathrm{BC}_{1 \mathrm{~b}}$ & $F_{1 b}$ & $F_{1 w}$ & $F_{2 b}$ & $\mathrm{~F}_{2 \mathrm{w}}$ & $\mathrm{BC}_{1 \mathrm{w}}$ & WSS & $F$ & $P$ \\
\hline Seed length (mm) & $\begin{array}{l}\text { Abidjan } \\
\text { Manfla }\end{array}$ & $\begin{array}{l}18.3 \pm 1.01^{\mathrm{a}} \\
18.4 \pm 0.91^{\mathrm{a}}\end{array}$ & $\begin{array}{l}17.61 \pm 1.17^{\mathrm{b}} \\
17.71 \pm 1.31^{\mathrm{b}}\end{array}$ & $\begin{array}{l}15.41 \pm 1.29^{\circ} \\
16.2 \pm 1.23^{c}\end{array}$ & $\begin{array}{l}14.7 \pm 1.17^{c} \\
16.14 \pm 0.97^{c}\end{array}$ & $\begin{array}{l}14.72 \pm 1.28^{d} \\
15.6 \pm 1.45^{d}\end{array}$ & $\begin{array}{l}14.24 \pm 1.66^{d} \\
15.57 \pm 1.72^{d}\end{array}$ & $\begin{array}{l}13.23 \pm 1.10^{\mathrm{e}} \\
14.49 \pm 1.34^{\mathrm{e}}\end{array}$ & $\begin{array}{l}11.37 \pm 0.75^{f} \\
12.17 \pm 0.68^{f}\end{array}$ & $\begin{array}{l}228.27 \\
142.94\end{array}$ & $\begin{array}{l}<0.001 \\
<0.001\end{array}$ \\
\hline Seed width (mm) & $\begin{array}{l}\text { Abidjan } \\
\text { Manfla }\end{array}$ & $\begin{array}{l}10.36 \pm 0.29^{\mathrm{a}} \\
10.52 \pm 0.74^{\mathrm{a}}\end{array}$ & $\begin{array}{l}9.2 \pm 0.71^{b} \\
9.26 \pm 0.87^{b}\end{array}$ & $\begin{array}{l}7.99 \pm 0.95^{c} \\
8.01 \pm 1.12^{c}\end{array}$ & $\begin{array}{l}7.6 \pm 0.67^{c} \\
7.98 \pm 0.62^{c}\end{array}$ & $\begin{array}{l}6.94 \pm 0.87^{d} \\
7.36 \pm 1.08^{d}\end{array}$ & $\begin{array}{l}7.18 \pm 1.28^{d} \\
7.39 \pm 1.54^{d}\end{array}$ & $\begin{array}{l}5.54 \pm 0.95^{\mathrm{e}} \\
6.09 \pm 1.14^{\mathrm{e}}\end{array}$ & $\begin{array}{l}4.13 \pm 0.36^{f} \\
4.09 \pm 0.33^{f}\end{array}$ & $\begin{array}{l}189.39 \\
230.72\end{array}$ & $\begin{array}{l}<0.001 \\
<0.001\end{array}$ \\
\hline Fresh seed mass (g) & $\begin{array}{l}\text { Abidjan } \\
\text { Manfla }\end{array}$ & $\begin{array}{l}53.91 \pm 9.92^{\mathrm{a}} \\
54.06 \pm 9.74^{\mathrm{a}}\end{array}$ & $\begin{array}{l}46.32 \pm 19.91^{b} \\
48.04 \pm 17.72^{b}\end{array}$ & $\begin{array}{l}45.08 \pm 10.84^{\mathrm{bc}} \\
45.91 \pm 11.01^{\mathrm{bc}}\end{array}$ & $\begin{array}{l}42.02 \pm 0.17^{\circ} \\
42.45 \pm 10.01^{\circ}\end{array}$ & $\begin{array}{l}31.85 \pm 17.45^{d} \\
37.34 \pm 14.68^{d}\end{array}$ & $\begin{array}{l}28.9 \pm 20.67^{d} \\
33.88 \pm 19.96^{\text {de }}\end{array}$ & $\begin{array}{l}29.33 \pm 10.91^{\mathrm{d}} \\
30.61 \pm 13.10^{\mathrm{e}}\end{array}$ & $\begin{array}{l}22.05 \pm 4.92^{f} \\
25.47 \pm 6.23^{f}\end{array}$ & $\begin{array}{l}35 \\
34.63\end{array}$ & $\begin{array}{l}<0.001 \\
<0.001\end{array}$ \\
\hline Dry seed mass (g) & $\begin{array}{l}\text { Abidjan } \\
\text { Manfla }\end{array}$ & $\begin{array}{l}27.35 \pm 5.92^{\mathrm{a}} \\
29.48 \pm 7.31^{\mathrm{a}}\end{array}$ & $\begin{array}{l}22 \pm 11.88^{b} \\
25.08 \pm 9.93^{b}\end{array}$ & $\begin{array}{l}24.31 \pm 5.47^{b} \\
25.13 \pm 5.94^{b}\end{array}$ & $\begin{array}{l}22.66 \pm 5.01^{b} \\
25.12 \pm 5.48^{b}\end{array}$ & $\begin{array}{l}13.87 \pm 9.74^{c} \\
20.39 \pm 10.43^{c}\end{array}$ & $\begin{array}{l}13.86 \pm 8.45^{\circ} \\
19.82 \pm 8.45^{\circ}\end{array}$ & $\begin{array}{l}9.81 \pm 5.29^{d} \\
14.79 \pm 6.29^{d}\end{array}$ & $\begin{array}{l}6.75 \pm 1.52^{\mathrm{e}} \\
9.97 \pm 3.47^{\mathrm{e}}\end{array}$ & $\begin{array}{l}49.42 \\
35.18\end{array}$ & $\begin{array}{l}<0.001 \\
<0.001\end{array}$ \\
\hline Hundred seeds mass (g) & $\begin{array}{l}\text { Abidjan } \\
\text { Manfla }\end{array}$ & $\begin{array}{l}16.78 \pm 1.53^{\mathrm{a}} \\
17.7 \pm 3.77^{\mathrm{a}}\end{array}$ & $\begin{array}{l}13.85 \pm 2.53^{\mathrm{b}} \\
14.76 \pm 2.92^{\mathrm{b}}\end{array}$ & $\begin{array}{l}9.76 \pm 2.26^{c} \\
11.52 \pm 2.12^{c}\end{array}$ & $\begin{array}{l}9.54 \pm 1.92^{\circ} \\
11.85 \pm 1.24^{\circ}\end{array}$ & $\begin{array}{l}8.2 \pm 2.08^{d} \\
10.43 \pm 2.29^{d}\end{array}$ & $\begin{array}{l}7.99 \pm 3.20^{d} \\
10.42 \pm 3.09^{d}\end{array}$ & $\begin{array}{l}5.69 \pm 2.42^{\mathrm{e}} \\
7.2 \pm 2.20^{\mathrm{e}}\end{array}$ & $\begin{array}{l}3.5 \pm 0.35^{f} \\
4.52 \pm 0.59^{f}\end{array}$ & $\begin{array}{l}293.38 \\
185.87\end{array}$ & $\begin{array}{l}<0.001 \\
<0.001\end{array}$ \\
\hline Seed integument thickness & $\begin{array}{l}\text { Abidjan } \\
\text { Manfla }\end{array}$ & $\begin{array}{l}36.64 \pm 4.00^{\mathrm{a}^{*}} \\
37.07 \pm 4.11^{\mathrm{a}}\end{array}$ & $\begin{array}{l}34.89 \pm 8.09^{b} \\
35.03 \pm 7.92^{b}\end{array}$ & $\begin{array}{l}29.46 \pm 5.59^{c} \\
32.57 \pm 4.98^{c}\end{array}$ & $\begin{array}{l}28.33 \pm 3.94^{c} \\
32.39 \pm 4.44^{c}\end{array}$ & $\begin{array}{l}24.23 \pm 6.87^{d} \\
28.69 \pm 8.30^{d}\end{array}$ & $\begin{array}{l}23.86 \pm 10.9^{d} \\
27.51 \pm 9.60^{d}\end{array}$ & $\begin{array}{l}19.64 \pm 9.23^{e} \\
19.04 \pm 7.75^{e}\end{array}$ & $\begin{array}{l}18.37 \pm 3.50^{\mathrm{e}} \\
18.84 \pm 3.79^{\mathrm{e}}\end{array}$ & $\begin{array}{l}79.35 \\
58.92\end{array}$ & $\begin{array}{l}<0.001 \\
<0.001\end{array}$ \\
\hline
\end{tabular}

Means with same letters along rows are not significantly different $(P<0.05)$ 


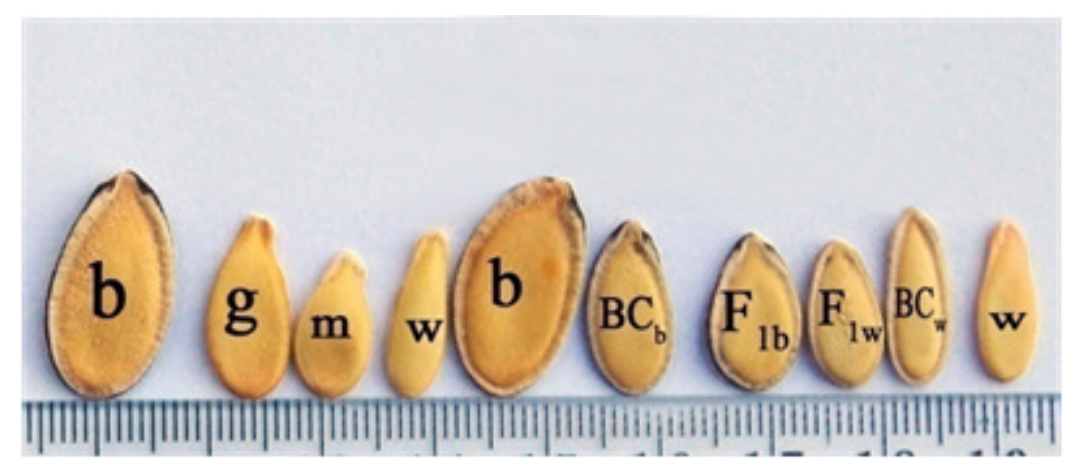

Figure 1. Parental (band $w$ ) and hybrids seeds. $b$ : bebu, w: Wlêwlê Small Seed, $F_{1 w}: F_{1}$ from

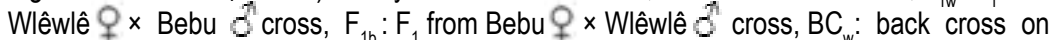
WSS cuitıvar; $\mathrm{BC}_{\mathrm{b}}$ : back cross on Bebu cultivar, g: recombinant form which look like "wlêwlêbig seed", m: recombinant form which look like "wlêwlêmedium seed"

TABLE 2. Mahalanobis genetic distance between populations and asterisk steady statistical test on Manfla (Abidjan)

\begin{tabular}{|c|c|c|c|c|c|}
\hline Populations* & Bebu & WSS & $\mathrm{BC}_{1 \mathrm{w}}$ & $\mathrm{BC}_{1 \mathrm{~b}}$ & $\mathrm{~F}_{2 \mathrm{w}}$ \\
\hline WSS & $67.45^{\star \star \star}\left(72.35^{\star \star \star}\right)$ & & & & \\
\hline $\mathrm{BC}_{1 \mathrm{w}}$ & $34.06^{\star \star \star}\left(44.28^{\star \star \star}\right)$ & $6.18^{\star \star \star}\left(4.62^{\star \star \star}\right)$ & & & \\
\hline $\mathrm{BC}_{1 \mathrm{~b}}^{\mathrm{iw}}$ & $4.47^{\star \star \star}\left(6.06^{\star \star \star}\right)$ & $30.61^{* * *}\left(40.93^{* * *}\right)$ & $15.28^{\star \star \star}\left(21.30^{\star \star \star}\right)$ & & \\
\hline $\mathrm{F}_{2 \mathrm{w}}{ }^{16}$ & $21.34^{\star \star \star}\left(23.22^{\star \star \star}\right)$ & $15.40^{\star \star \star}\left(16.28^{\star * *}\right)$ & $2.77^{\star \star \star}\left(4.98^{\star \star \star}\right)$ & $4.23^{\star \star \star}\left(7.06^{\star \star \star}\right)$ & \\
\hline $\mathrm{F}_{1 \mathrm{w}}^{2 \mathrm{w}}$ & $19.97^{\star \star \star}\left(24.53^{\star \star \star}\right)$ & $16.99^{\star \star \star}\left(20.51^{\star \star \star}\right)$ & $6.33^{\star \star *}\left(7.45^{\star \star *}\right)$ & $3.84^{\star \star \star}\left(7.19^{\star \star \star}\right)$ & $1.83^{* \star *}\left(1.13^{* \star *}\right)$ \\
\hline
\end{tabular}

WSS: Wlêwlê Small Seed, $\mathrm{BC}_{1 w}$ : back cross on WSS cultivar using $\mathrm{F}_{1 \mathrm{w}}$ male flowers, $\mathrm{BC}_{16}$ : back cross on Bebu cultivar using

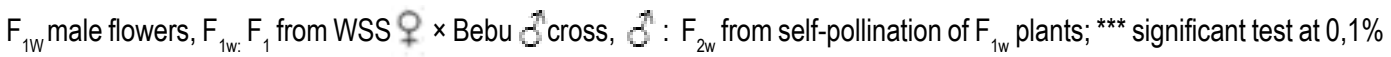

TABLE 3. Individuals repartition according to population on both locations

\begin{tabular}{|c|c|c|c|c|c|c|c|c|}
\hline \multirow[t]{2}{*}{ Locations } & \multirow[t]{2}{*}{ Families } & \multirow{2}{*}{$\begin{array}{l}\text { Percentage of } \\
\text { well classified } \\
\text { individuals (\%) }\end{array}$} & \multicolumn{6}{|c|}{ Number of well classified individuals per population } \\
\hline & & & Bebu & WSS & $\mathrm{BC}_{1 \mathrm{w}}$ & $\mathrm{BC}_{1 \mathrm{~b}}$ & $F_{2 w}$ & $F_{1 w}$ \\
\hline \multirow[t]{7}{*}{ Manfla (Abidjan) } & Bebu & $54.55(69.05)$ & $24(29)$ & $0(0)$ & $0(0)$ & $16(12)$ & $4(3)$ & $0(0)$ \\
\hline & WSS & $96.49(72.73)$ & $0(0)$ & $55(24)$ & $2(9)$ & $0(0)$ & $5(5)$ & $0(0)$ \\
\hline & $\mathrm{BC}_{1 w}$ & $57.01(88.00)$ & $0(0)$ & $9(2)$ & $61(176)$ & $4(5)$ & $33(18)$ & $6(4)$ \\
\hline & $\mathrm{BC}_{1 \mathrm{~b}}^{1 \mathrm{w}}$ & $80.35(90.48)$ & $5(4)$ & $0(0)$ & $1(2)$ & $184(247)$ & $31(16)$ & $8(6)$ \\
\hline & $\mathrm{F}_{2 \mathrm{w}}{ }^{10}$ & $66.27(51.06)$ & $2(2)$ & $2(3)$ & $28(39)$ & $38(36)$ & $165(62)$ & $14(14)$ \\
\hline & $F_{1 w}^{2 w}$ & $41.25(55.75)$ & 0 & 0 & $4(3)$ & $8(7)$ & $39(40)$ & $33(63)$ \\
\hline & Total & $68.15(74.01)$ & $31(35)$ & $66(29)$ & $96(229)$ & $250(307)$ & $277(144)$ & $61(87)$ \\
\hline
\end{tabular}

WSS = Wlêwlê Small Seed, $\mathrm{BC}_{1 \mathrm{w}}=$ back cross on WSS cultivar using $\mathrm{F}_{1 \mathrm{w}}$ male flowers, $\mathrm{BC}_{1 \mathrm{~b}}=$ back cross on Bebu cultivar using $F_{1 W}$ male flowers, $F_{1 w:} F_{1}$ from WSS $q \times$ Bebu $\delta^{n}$ cross, $\delta^{n}=F_{2 w}$ from self-pollination of $F_{1 w}$ plants 
experimental site, and $77.50 \%$ for Manfla. This factor, which expressed more than $77 \%$ of total variability, had been used for result interpretation. Correlation matrix between characters and axis 1 and 2 showed that factor 1 was defined by all variables on both sites (Table 5). Seed characters were positively correlated to axis 1 . This axis can be used as production axis.

The projection of the individuals in plan 1 and 2 showed 3 main groups on both experimental sites (Fig. 2). The first group contained individuals, which looked like WSS individuals. It regrouped all WSS individuals and some $\mathrm{BC}_{1 \mathrm{w}}$ and $F_{2}$ individuals. The second group contained hybrid individuals, which looked like $\mathrm{F}_{1}$ hybrids. It regrouped all $\mathrm{F}_{1}$ individual, some $\mathrm{BC}_{1 \mathrm{w}}, \mathrm{BC}_{1 \mathrm{~b}}$ and $\mathrm{F}_{2}$ individuals. The third group contained individuals which looked like Bebu individuals. It regrouped all Bebu individuals and some $\mathrm{BC}_{1 \mathrm{~b}}$ and $\mathrm{F}_{2}$ individuals.

PCA revealed that Bebu cultivar was different from WSS cultivar. $F_{1}$ hybrids were intermediate to both parents; while $\mathrm{F}_{2}$ population had a wide individual dispersion. Those individuals were present in all groups. $\mathrm{BC}_{1 \mathrm{w}}$ individuals were close to the ones of WSS; while $\mathrm{BC}_{1 \mathrm{~b}}$ individuals were close to the ones of Bebu.
Bebu was more heterogeneous than WSS because Bebu's genetic variance was higher than one of WSS (Table 6). Generally, genetic variance from $\mathrm{F}_{2}$ population was higher than for $\mathrm{BC}_{1}$ population, which was also higher than those of parental and $F_{1}$ populations. This shows that, $F_{2}$ population was more heterogeneous, while parental and $F_{1}$ populations were more homogeneous.

Phenotypic $\left(\sigma^{2}(\mathrm{P})\right)$, environment $\left(\sigma^{2}(\mathrm{E})\right)$, genotypic $\left(\sigma^{2}(\mathrm{G})\right)$ and additive $\left(\sigma^{2}(\mathrm{~A})\right)$ variances, large and strict heritabilities and genetic advance were grouped in Table 7. Environmental variance was weak; while phenotypic, genotypic and additive variances were high. Environment effects on seed variability were very weak, which led to high heritabilities (large and strict) $\left(\mathrm{H}^{2}>\right.$ 0.50 and $h^{2}>0.50$ ) for all characters on both site. More than $50 \%$ of seed variability between populations was imputable to genetic variance. All of these characters can be transferred exactly to offsprings whatever the environment percentage. Genetic advance was high for seed mass on both sites.

The number of effective factors for each character on both sites, is summrised in Table 8 . The effective factor estimation method used

TABLE 4. Matrice of principales composantes on both experimentales sites

\begin{tabular}{lccccc}
\hline Locations & Axes & Value & Variances & Cumulated value & Cumulated Variances \\
\hline Manfla (Abidjan) & 1 & $8.52(8.50)$ & $77.50(77.31)$ & $8.52(8.50)$ & $77.50(77.31)$ \\
& 2 & $0.63(0.78)$ & $5.73(5.73)$ & $9.15(9.28)$ & $83.24(84.43)$ \\
\hline
\end{tabular}

TABLE 5. Correlation matrix between traits and axes at Manfla (Abidjan)

\begin{tabular}{lrr}
\hline Traits & Factor 1 & Factor 2 \\
\hline Seed length (SL) & $0.87(0.96)$ & $-0.32(0.24)$ \\
Seed width (SW) & $0.91(0.94)$ & $-0.37(0.30)$ \\
Fresh seed mass (FSM) & $0.93(0.90)$ & $0.18(0.00)$ \\
Dry seed mass (DSM) & $0.94(0.95)$ & $0.17(-0.06)$ \\
Hundred seeds mass (HSM) & $0.93(0.93)$ & $-0.30(0.31)$ \\
Percentage of seed integument (PSI) & $0.90(0.87)$ & $-0.25(0.22)$ \\
Variance expliquée (\%) & $22(14)$ & $17(-37)$ \\
\hline
\end{tabular}




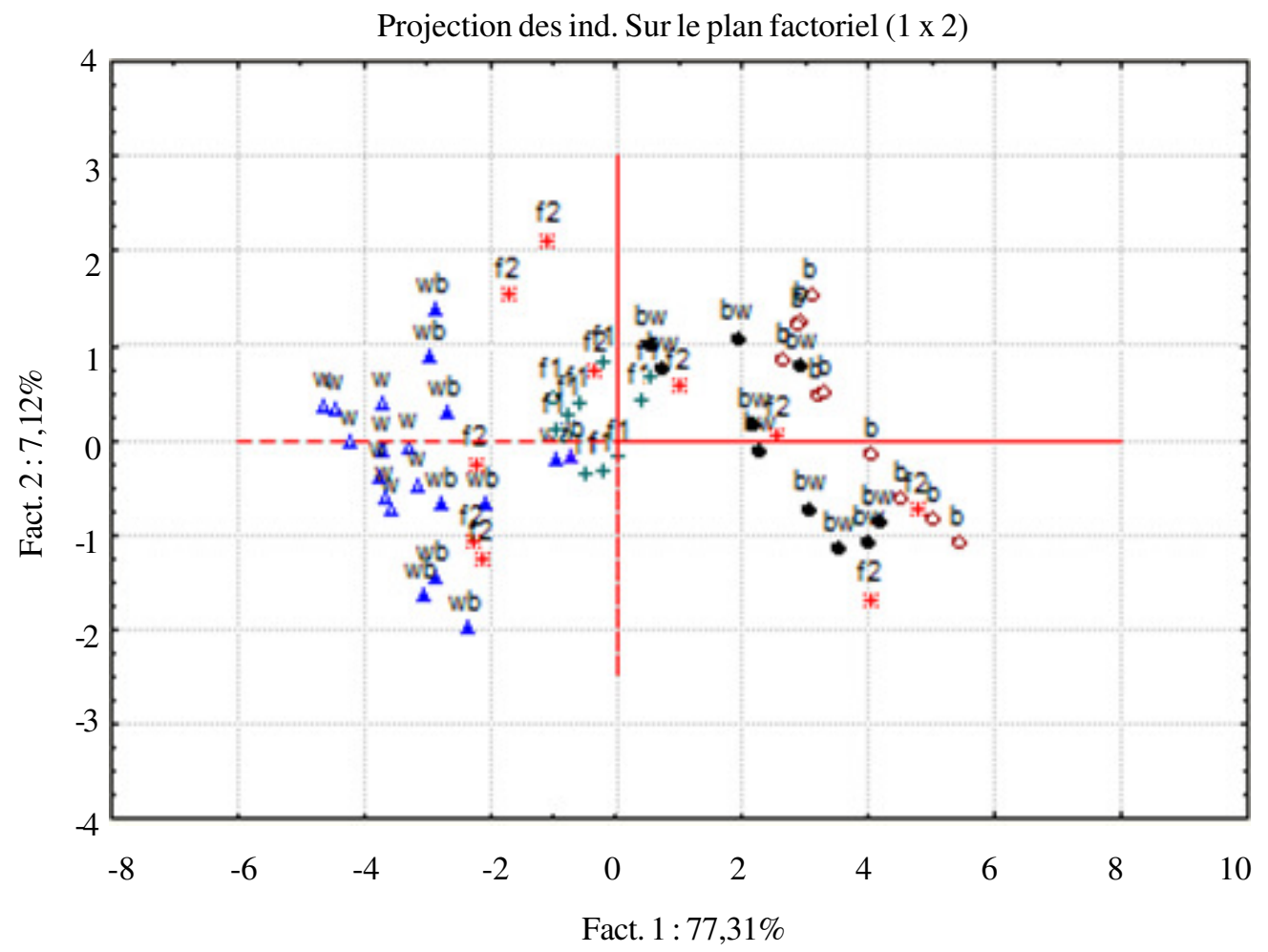

Figure 2. Individuals repartition in first plan of PCA. Individuals repartition is the same on both sites. So, here we represent for one site (Abidjan). b: bebu, WSS: Wlêwlê Small Seed, wb: back cross on WSS cultivar using $F_{1 W}$ male flowers, bw: back cross on Bebu cultivar using $F_{1 W}$ male flowers, $F_{1} F_{1}$ from WSS $q \times$ Bebu $\delta^{\prime}$ cross, $\delta^{\hbar}: F_{2}$ from self-pollination of $F_{1}$ plants.

provided similar result on both experimentation sites. The effective factor calculated was statically identical to unity (1) for spawn and dry seed mass, and seed integument thickness. Thus, these traits were governed by only one gene pair. However, for 100 seeds mass, length and width seeds, effective factor calculated turn around three. These characters were governed by at least 3 effective genes.

\section{DISCUSSION}

Seed size, seed mass and seed integument thickness varied significantly among populations (Table 1). This inter-population variability had been confirmed by genetic distance and PCA tests and must be amputated to difference between parental seeds. PCA revealed a difference between Bebu and WSS seeds. All analysed parameters discriminated them clearly and showed seed genetic diversity in $C$. lanatus as had mentioned by Guner and Wehner (2004) and Gusmini and Wehner (2005).

This phenotypic difference between parental seeds increased the heterozygote index in their offsprings and yielded different seeds types. This heterozygote index produced wide seed variability in $\mathrm{BC} 1$ and especially in $\mathrm{F}_{2}$ where there was character segregation according to Mendel laws. Gusmini et al. (2004) also observed seeds character segregation in $\mathrm{BC}_{1}$ and $\mathrm{F}_{2}$ populations during crossing between two $C$. lanatus cultivars: Charleston Gray 3 PI 560006 and Calhoun Gray 3PI 490383w.

This character segregation was the evidence that some individuals can be identical to one of the parents; while others must be recombinants exhibiting different proportions of parental characters (Vedele and Loudet, 2001). So, the projection of the individuals in first PCA plan confirmed ANOVA test, and permitted to class all populations' seed into 3 main groups on both 
TABLE 6. Phenotypic variances for seeds traits

\begin{tabular}{|c|c|c|c|c|c|c|c|}
\hline Locations & Families & $S L$ & SW & FSM & DSM & HSM & PSI \\
\hline \multirow[t]{6}{*}{ Manfla (Abidjan) } & Bebu & $0.83(1.02)$ & $0.55(0.08)$ & $94.84(98.44)$ & $53.49(35.01)$ & $14.25(2.34)$ & $16.87(15.98)$ \\
\hline & WSS & $0.47(0.56)$ & $0.11(0.13)$ & $38.75(24.20)$ & $12.01(2.31)$ & $\begin{array}{r}0.35 \\
-0.13\end{array}$ & $14.34(12.25)$ \\
\hline & $\mathrm{BC}_{1 \mathrm{~b}}$ & $1.73(1.37)$ & $0.76(0.50)$ & $314.15(396.40)$ & $98.63(141.10)$ & $\begin{array}{r}8.51 \\
-6.39\end{array}$ & $62.69(65.50)$ \\
\hline & $\mathrm{BC}_{1 \mathrm{w}}$ & $1.79(1.20)$ & $1.31(0.91)$ & $171.63(119.10)$ & $39.63(127.99)$ & $\begin{array}{r}4.83 \\
-5.86\end{array}$ & $60.13(85.29)$ \\
\hline & $\mathrm{F}_{2 \mathrm{~W}}$ & $2.95(2.75)$ & $2.36(1.65)$ & $398.30(427.07)$ & $109.19(219.97)$ & $9.56(10.23)$ & $92.22(118.79)$ \\
\hline & $\mathrm{F}_{1 \mathrm{~W}}^{2 \mathrm{w}}$ & $0.94(1.37)$ & $0.39(0.45)$ & $100.27(103.42)$ & $30.04(25.14)$ & $\begin{array}{r}1.53 \\
-3.69\end{array}$ & $19.71(15.51)$ \\
\hline
\end{tabular}

FSM = Fresh seed mass, DSM = Dry seed mass, HSM = hundred seeds mass, PSI = Percentage of seed integument: $S L=$ Seed length, SW = Seed width, WSS = Wlêwlê Small Seed, BC $=$ back cross on WSS cultivar using $F_{1 w}$ male flowers, $B_{1 b}=$ back cross on Bebu cultivar using $F_{1 w}$ male flowers, $F_{1 w} F_{1}$ from WSS $Q \times$ Bebu $\delta^{2}$ cross, $\delta$ : $F_{2 w}$ from self-pollination of

$\mathrm{F}_{1 \mathrm{w}}$ plant

TABLE 7. Variance, heritability and genetic gain to selection estimates for families for seeds traits

\begin{tabular}{|c|c|c|c|c|c|c|c|c|c|}
\hline \multirow[t]{2}{*}{ Locations } & \multirow[t]{2}{*}{ Traits } & \multicolumn{4}{|c|}{ Variances } & \multicolumn{2}{|c|}{ Heritability } & \multicolumn{2}{|c|}{ Genetic advance } \\
\hline & & $\sigma^{2}(P)$ & $\sigma^{2}(E)$ & $\sigma^{2}(G)$ & $\sigma^{2}(\mathrm{~A})$ & $\mathrm{H}^{2}$ & $h^{2}$ & $5 \%$ & $10 \%$ \\
\hline \multirow[t]{6}{*}{ Manfla (Abidjan) } & $S L$ & $2.95(2.75)$ & $0.80(1.08)$ & $2.15(1.67)$ & $2.07(1.62)$ & $0.73(0.61)$ & $0.70(0.59)$ & $0.07(0.09)$ & $0.14(0.18)$ \\
\hline & SW & $2.36(1.65)$ & $0.36(0.28)$ & $2.01(1.37)$ & $1.66(1.09)$ & $0.85(0.83)$ & $0.70(0.66)$ & $0.09(0.07)$ & $0.17(0.14)$ \\
\hline & FSM & $398.30(427.07)$ & $83.53(82.37)$ & 314.77 (344.71) & $310.83(338.66)$ & $0.79(0.81)$ & $0.78(0.79)$ & $0.78(0.82)$ & $1.56(1.64$ \\
\hline & DSM & $109.19(219.97)$ & $31.40(21.90)$ & $77.79(198.07)$ & $70.11(170.85)$ & $0.71(0.90)$ & $0.64(0.78)$ & $0.38(0.58)$ & $0.77(1.15)$ \\
\hline & HSM & $9.56(10.23)$ & $4.42(2.46)$ & $5.15(7.77)$ & $4.78(6.81)$ & $0.54(0.76)$ & $0.50(0.66)$ & $0.09(0.13)$ & $0.19(0.26)$ \\
\hline & PSI & $92.22(118.79)$ & $17.66(14.81)$ & 74.56 (103.98) & $61.62(86.79)$ & $0.81(0.88)$ & $0.67(0.73)$ & $0.32(0.40)$ & $0.64(0.80)$ \\
\hline
\end{tabular}

FSM = Fresh seed mass, DSM = Dry seed mass, HSM = hundred seeds mass, $\mathrm{PSI}=$ Percentage of seed integument: $\mathrm{SL}=$ Seed length, $\mathrm{SW}=\mathrm{Seed}$ width

Phenotypic $(P)$, environmental $(E)$, genotypic $(G)$, additive $(A)$ variance effects, broad $\left(H^{2}\right)$ and narrow $\left(h^{2}\right)$ sense heritability and genetic advance $(G s)$ of each character were estimated from generation variances as follows (Warner, 1952; Wright, 1968) 
TABLE 8. Number of effective factor for each character on both sites

\begin{tabular}{lrrrrrr}
\hline & $\begin{array}{c}\text { Seed } \\
\text { length }\end{array}$ & $\begin{array}{c}\text { Seed } \\
\text { width }\end{array}$ & $\begin{array}{c}\text { Fresh } \\
\text { seed mass }\end{array}$ & $\begin{array}{c}\text { Dry } \\
\text { seed mass }\end{array}$ & $\begin{array}{c}\text { Hundred } \\
\text { seeds mass }\end{array}$ & $\begin{array}{c}\text { Percentage } \\
\text { of seed } \\
\text { integument }\end{array}$ \\
\hline Abidjan & 3.37 & 3.04 & 0.39 & 0.27 & 3.37 & 0.4 \\
Manfla & 2.94 & 2.97 & 0.34 & 0.6 & 2.7 & 0.57 \\
Mean & 3.16 & 3 & 0.37 & 0.44 & 3.04 & 0.49 \\
Number of effective factor & 3 & 3 & 1 & 1 & 3 & 1 \\
\hline
\end{tabular}

The number of effective factors $(\mathrm{N})$, an estimate of the genetic factors determining a quantitative trait (Mendelian genes or quantitative trait loci), was estimated as follows:

$$
N=\frac{D^{2}}{8\left(\sigma^{2}\left(F_{2}\right)-\sigma^{2}\left(F_{1}\right)\right.}
$$

Where: $D=$ numeric value from difference between mean (i) parental line: $D=\left(\mu_{\text {Bebu }}-\mu_{\text {wss }}\right)$

experimental sites (Fig. 2). In the parental types, plant individuals which were grouped with WSS produced numerous small light seeds per fruit, while individuals which were grouped with Bebu, produced few big and heavy seed per fruit. The recombinant type had intermediate seed characters to both parents like $\mathrm{F}_{1}$ hybrids.

Fulks et al. (1979) and Zamir (2001) also observed intermediate forms resulting from natural crosses between $C$. lanatus and its wild ancestor $C$. colocynthis. $\mathrm{F}_{2}$ individuals were present in all groups that this population had wide seeds dispersion because many seed types had been observed in this population (characters segregation according to Mendel laws). Nevertheless, majority of $\mathrm{F}_{1}$ and $\mathrm{F}_{2}$ hybrids looked more like WSS than Bebu according to evaluated genetic distances (Table 2). This result must be imputed to considered cross type $\left(W S S_{@ \&} \mathrm{x}\right.$ $B e b u_{\mathrm{B} \&}$ ) during genetic distance evaluation. So, many of $F_{1}$ and $F_{2}$ progenies looked like their female parents. However, statistical analyses showed that cross direction did not affect $F_{1}$ and $F_{2}$ seeds because $F_{1 b}$ and $F_{1 w}$, and $F_{2 b}$ and $F_{2 w}$ were statically similar. On the other hand, cross direction affected $\mathrm{BC}_{1}$ seeds characters due to the maternal effect. Observations showed that Bebu and $\mathrm{BC}_{1 \mathrm{~b}}$ and WSS and $\mathrm{BC}_{1 \mathrm{w}}$ were similar genetically (Fig. 2). So, backcrossing on Bebu yields few, big and heavy seeds, with thick integument; while backcrossing on WSS exhibited numerous seeds, small and light seeds with thin integument (Table 1).
Phenotypical similarity between $\mathrm{BC}_{1}$ hybrids and their maternal parents, suggests the existence of sufficient parental genes compared with $F_{1}$ hybrids during backcrossing, in expression of examined characters. These results were the evidence that cross direction is an important factor for $C$. lanatus genetic breeding. Variability in quantitative and qualitative character expressions according to cross direction had been already observed in C. lanatus (Henderson et al., 1998; Gusmini et al., 2004).

These observations suggest that Bebu and $\mathrm{BC}_{1 \mathrm{~b}}$, and, WSS and $\mathrm{BC}_{1 \mathrm{w}}$ were similar genetically. These genetic distances and statistical test between populations confirmed ANOVA results and certified wide variability between populations on both site. Similar results were reported by Bodzon (2004), Akbar et al. (2008) and Waqar-UlHaq et al. (2008). Thus, all characters examined admitted high large and strict heritabilities, irrespective of experimental site (Table 7). This suggests that environment effects are less important than genetic effects. More than 50\% of character variability observed in populations were bound to genetic variability. This high heritability confirmed the high difference between both selected parents (Singh and Westermann, 2002). For all of these characters, genotypic and additive variances were widely superior to environmental variance on both sites. In addition, high heritability of seed mass was proportional to high genetic advance on both sites. Individuals' selection according to seed mass, 
irrespective of environment could be profitable to cultivar breeding. Breeders can choose plants which yield heavy seeds. Similar observations were made on fruit characters (Gusmini and Wehner, 2005; Kumar, 2009).

The number of effective factors which controlled each character was also evaluated (Table 8). It permitted to the observation that spawn and dry seed mass and seed integument thickness had been controlled by one effective factor; these characters were monogenic. One hundred seed mass, seed length and width had been controlled by 3 effectives factors; these characters were polygenic. The first group was governed by one allelic pair, while the second was governed by at least 3 effective genes. Several studies showed that number of effective factors, which governed each character, varied considerably according to crosses and experimental sites (Gusmini and Wehner, 2005; Kumar, 2009; Lou, 2009).

\section{CONCLUSION}

This study show large genetic variability between populations (parental and hybrid) seed. These populations were classed to 3 main groups: two parental types including each parental type with its offspring maternal backcross and some $\mathrm{F}_{2}$ individuals and one recombinant type including all $\mathrm{F}_{1}$ individuals and some $\mathrm{BC}_{1 \mathrm{~b}}, \mathrm{BC}_{1 \mathrm{w}}$ and $\mathrm{F}_{2}$ individuals.

Phenotypic similarity between $\mathrm{BC}_{1}$ hybrids and their maternal parents suggests existence of sufficient parental genes than in $\mathrm{F}_{1}$ hybrids during backcrossing in expression of examined characters. These results are the evidence that cross direction is an important factor for $C$. lanatus genetic breeding.

All characters examined, admitted a high large and strict heritabilities, suggesting that environment effects are less important than genetic effects. None of these characters were monogenic while others were polygenic.

\section{REFERENCES}

Abdel, C.G. and Bamerni, K.A.A. 2011. Effect of pre-planting land flooding durations on growth, yield and anatomical parameters of three Watermelon [Citrullus lanatus(Thunb.) Matsum.] Cultivars. American Journal of Experimental Agriculture 1:187-213.

Achigan-Dako, E.G., Fagbemissi, R., Avohou, H.T., Vodouhe, R.S., Coulibaly, O. and Ahanchede, A. 2008. Importance and practices of Egusi crops (Citrullus lanatus (Thunb.) Matsum. and Nakai, Cucumeropsis mannii Naudin and Lagenaria siceraria (Molina) Standl. in sociolinguistic areas in Benin. Biotechnology, Agronomy, Society and Environment 12:393-403.

Adjoumani, K., Kouonon, L.C., Akaffou, D.S. and Djè, Y. 2012. Diversité variétale chez l'espèce cultivée Citrullus lanatus (Matsumara et Nakai) et opportunité d'amélioration génétique des cultivars. European Journal of Scientific Research 67:564-579.

Akbar, M., Shakoor, M.S., Hussain, A. and Sarwar, M. 2008. Evaluation of maize 3-way crosses through genetic variability, broad sens heritability, characters association and path analysis. Journal Agriculture Research 46:39-45.

Bodzon, Z. 2004. Correlations and heritability of the characters determining the seed yield of the long-raceme alfalfa (Medicago sativa $\mathrm{L}$.). Journal Apple Genetic 45: 49-59.

Dos Santos, M.H., Rodrigues, R., Gonçalves, L.S.A., Sudré, C.P. and Pereira, M.G. 2012. Agrobiodiversity in Cucurbita spp. Landraces collected in Rio de Janeiro assessed by molecular markers. Crop Breeding and Applied Biotechnology 12:96103.

Falconer, D.S. 1989. Introduction to quantitative genetics. Ltd RC (Ed). pp. 129-185.

Fulks, B.K., Scheerens, J.C. and Bemis, W.P. 1979. Natural hybridization of two Citrullus species. Journal of Heredity 70:214-215.

Guner, N. and Wehner, T.C. 2004. The genes of watermelon. HortScience 39: 1175-1182.

Gusmini, G. and Wehner, T.C. 2005. Genes determining rind patter inheritance in watermelon: Areview. HortScience 40:19281930.

Gusmini, G., Wehner, T.C. and Jarret, R.L. 2004. Inheritance of egusi type in watermelon. Journal of Heredity 95:268-270. 
Henderson, W.R., Scott, G.H. and Wehner, T.C. 1998. Interaction of flesh color genes in watermelon. Journal of Heredity 89:50-53.

Kouassi, N.J. and Zoro, Bi, I.A. 2009. Effect of sowing density and seedbed type on yield and yield components in bambara groundnut (Vigna subterranea) in woodland savana of Côte d'Ivoire. Experimental Agriculture 46:99-110.

Kumar, R. 2009. Inheritance of fruit yield and other horticulturally important traits in watermelon [Citrullus lanatus (Thunb.) Matsumara \& Nakai]. PhD. North Carolina State University, USA. 182p.

Kumar, R., Wehner, T.C. 2011. Natural outcrossing in watermelon: A Review. Cucurbit Genetics Cooperative Report 34: 42-43.

Lou, L. 2009. Inheritance of fruit characteristics in watermelon [Citrullus lanatus (Thunb.) Matsum. \& Nakai]. PhD. Thesis, North Carolina State University, USA.

Nerson, H. 2007. Seed Production and Germinability of Cucurbit Crops. Seed Science and Biotechnology 1:1-10.

SAS 2006. SAS Enterprise Guide. Release 4.01 edn. Cary, NC: SAS Institute, USA.

Singh, S.P. and Westermann, D.T. 2002. Crop breeding, genetics and cytologya: Single dominant gene controlling resistance to soil zinc deficiency in common bean. Crop Science 42:1071-1074

Tazeen, M., Nadia, K. and Farzana, N.N. 2009. Heritability, phenotypic correlation and path coefficient studies for some agronomic characters in synthetic elite lines of wheat.
Journal of Food, Agriculture and Environment 7:278-282.

Vedele, F. and Loudet, O. 2001.Un exemple de la dissection génétique d'un caractère complexe: lignées recombinantes et recherche de QTLs. INRA.pp. 1-5.

Waqar-Ul-Haq, Malik,M.F., Rashid, M., Munir, M. and Akram, Z. 2008. Evaluation and estimation of heritability and genetic advancement for yield related attributes in wheat lines. Pakistan Journal of Botany 40: 1699-1702.

Warner, J.N. 1952. A method for estimating heritability. Agronomy Journal 44: 427-430.

Wright, S. 1968. The genetics of quantitative variability, In: Wright, S. (Ed.). Evolution and genetics of populations. University of Chicago Press. pp. 373-420.

Zamir, D. 2001. Improving plant breeding with exotic genetic libraries. Genetics 2: 983-989.

Zoro Bi, I.A., Koffi, K.K. and Djè, Y. 2006. Caractérisation botanique et agronomique de trois espèces de cucurbites consommées en sauce en Afrique de l'Ouest: Citrullus sp., Cucumeropsis manii Naudin et Lagenaria siceraria (Molina) Standl. Biotechnology, Agronomy, Society and Environment 7:189199.

Zoro Bi, I.A., Koffi., K.K. and Djè, Y. 2003. Caractérisation botanique et agronomique de trois espèces de cucurbites consommées en sauce en Afrique de l'Ouest: Citrullus sp., Cucumeropsis manii Naudin et Lagenaria siceraria (Molina) Standl. Biotechnology, Agronomy, Society and Environment 7: 189199. 